De Miguel Álvarez, Laura; Rúas Ruiz-de Infante, Jaime; Ríos Aguilar, Sergio; González Crespo, Rubén El Museo Virtual ESIT como recurso para la enseñanza-aprendizaje en áreas de Diseño y Tecnología IV Congreso INTERNACIONAL DE INVESTIGACIÓN EN ARTES VISUALES ANIAV 2019 IMAGEN [N] VISIBLE]

http://dx.doi.org/10.4995/ANIAV.2019.9000

De Miguel Álvarez, Laura.

Escuela Superior de Ingeniería y Tecnología (ESIT). Universidad Internacional de la Rioja.

Rúas Ruiz-de Infante, Jaime.

Escuela Superior de Ingeniería y Tecnología (ESIT). Universidad Internacional de la Rioja.

Ríos Aguilar, Sergio.

Escuela Superior de Ingeniería y Tecnología (ESIT). Universidad Internacional de la Rioja.

González Crespo, Rubén.

Escuela Superior de Ingeniería y Tecnología (ESIT). Universidad Internacional de la Rioja.

\title{
El Museo Virtual ESIT como recurso para la enseñanza- aprendizaje en áreas de Diseño y Tecnología
}

\section{ESIT Virtual Museum as a resource for teaching-learning in Technology and Design areas}

TIPO DE TRABAJO: Comunicación Virtual

PALABRAS CLAVE

Diseño, Tecnología, Arte, Museo, Creatividad.

KEY WORDS

Design, Technology, Art, Museum, Creativity.

RESUMEN

El Museo Virtual ESIT, es producto de un Proyecto de Innovación Educativa (PIE) ocupado de promover acciones bajo la perspectiva STEAM, apoyándose en la metodología Learning by doing en áreas de ingeniería, para que la creatividad y la tecnología se complementen en la búsqueda y aplicación de una solución funcional, real y bella. Y que, además, coloca al estudiante en el centro de su experiencia formativa en la que también pueda experimentar situaciones reales a la hora de presentarse a convocatorias de concurso, oportunidades laborales altamente competitivas o convocatorias públicas o privadas que concedan financiación para el desarrollo de un proyecto determinado.

El proyecto Museo Virtual ESIT es un proyecto, a través del cual, se genera un espacio arquitectónico utilizando la herramienta Unity, que hace posible centrarse en la creación de un metamodelo de mundo virtual. En este caso se emula las distintas salas de un museo o galería de arte, donde exponer las soluciones producidas por la actividad formativa desarrolla en el área de Diseño de la Escuela Superior de Ingeniería y Tecnología (ESIT) de la Universidad Internacional de la Rioja (UNIR).

\section{ABSTRACT}

The ESIT Virtual Museum is the product of an educational innovation project which offers some actions under the STEAM perspective, supporting on the "Learning by doing" methodology in engineering areas, so that creativity and technology complement each other in the search and application of functional, real and aesthetic solution. The student is placed at the center of their formative experience, 
in which they can also experience real situations in terms of appearing in competitive exams, highly competitive job opportunities or public or private calls that grant financing for the development of a specific project.

The ESIT Virtual Museum is an architectural space project with the Unity tool to develop a metamodel of the virtual world. In this case, the solutions produced by the training activity are developed in the Design area of the Higher School of Engineering and Technology (ESIT) of the International University of La Rioja (UNIR) are exposed in the different rooms of a museum or an art gallery.

\section{INTRODUCCIÓN}

La Comisión Europea, en su programa marco 2014-2020, dedica más de 13 millones de euros a subvencionar iniciativas que se dediquen a aumentar el atractivo de la educación científica y de las carreras científicas e impulsar el interés de los jóvenes en STEM, acrónimo en inglés que aglutina cuatro materias de orden académico: Science, Tecnology, Engineering y Mathematics. Pero en los últimos años se ha observado un ligero matiz diferenciador entre las acciones STEM del inicio de implantación de este modelo y las que se desarrollan actualmente. Los expertos en la materia, creen que estos cambios se deben a la inclusión en el ámbito educativo del fomento del pensamiento creativo y el trabajo práctico más basado en actividades competenciales. Así, cuando se conjugan habilidades artístico-creativas con el modelo STEM se ponen en valor soluciones en las que la innovación, el desarrollo de la imaginación y el pensamiento divergente, hacen que se produzca la incorporación en este modelo de la materia Arts, transformando su nombre de STEM a STEAM (Muñoz, 2015).

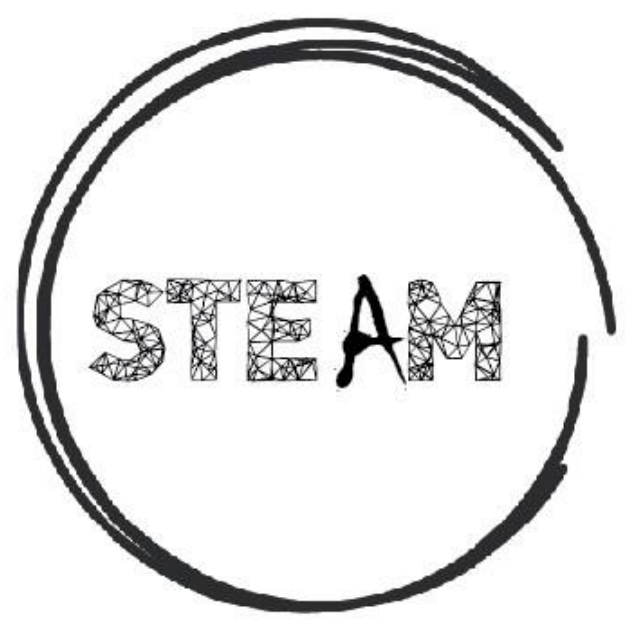

Figura 1: Producto visual sobre el concepto STEAM, 2019. Elaboración propia.

Gracias a esta metodología los estudiantes pueden aprender a través de proyectos que integran la aplicación de conocimientos de diferentes materias, entre las que se encuentran las humanidades (Arts), trabajando en la búsqueda de una solución que conecte el aprendizaje con una experiencia de la vida real (Johanson, 2018). Los estudiantes trabajan de forma integrada frente a un determinado reto a través de la ciencia, la tecnología y/o la creatividad en la toma de decisiones transversalmente a los objetivos a alcanzar del proyecto en cuestión, del mismo modo que lo hacen en su vida cotidiana.

En este paradigma surge el PIE Museo Virtual ESIT durante el curso académico 2017-2018, desarrollado por Personal Docente Investigador (PDI) de UNIR, con la intención de ofrecer a los estudiantes del área de Diseño la posibilidad de ampliar el marco de aplicación de los conocimientos que van adquiriendo en sus formaciones (de grado y máster) hacia propuestas que estén conectadas directamente con el ámbito profesional en formato de convocatorias competitivas de difusión del propio trabajo, a través de concursos, exposiciones y/o colaboraciones con profesionales del sector como, por ejemplo, Isidro Ferrer ${ }^{1}$.

\footnotetext{
${ }^{1}$ Para la última exposición realizada, inaugurada en marzo de 2019, se cuenta con la colaboración de este afamado diseñador cuyas obras más características están expuestas junto a las de los estudiantes seleccionados para la muestra.
} 


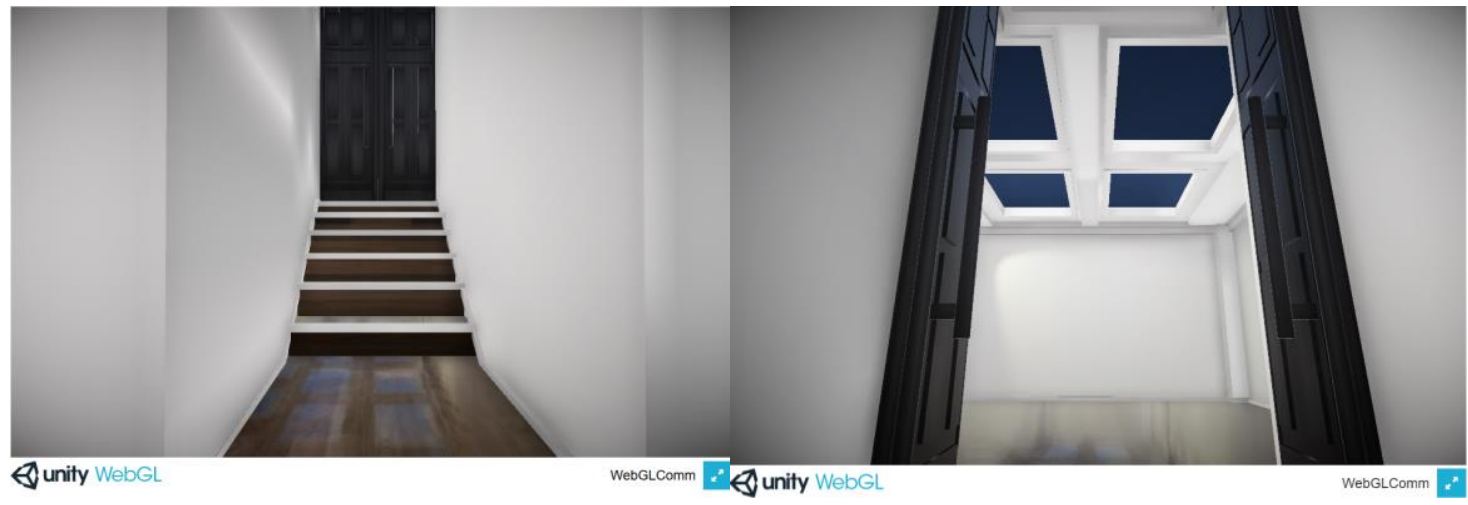

Figura 2 y 3: Capturas de pantalla del recorrido virtual para acceder al Museo Virtual ESIT, 2018. Proyecto Museo Virtual ESIT.

\section{La construcción de experiencias memorables en la presentación de acciones STEAM}

Aunque la "A" (Arts) de STEAM en realidad hace referencia al área de Humanidades, a lo largo de este proyecto se ha establecido también cierta relación con su traducción literal en español "Arte", ya que parte de los docentes de Diseño que desarrollan este proyecto provienen de esa área. Existe un pulso singular que provoca que personas que provienen del ámbito de las Bellas Artes más puras (pintura, restauración, escultura, grabado) se sumerjan en el fascinante mundo del diseño pues los procesos, procedimientos y métodos de creación artística ofrecen su posible traducción para la aplicación de la tecnología en el diseño, pero, como apuntan Haro y Alonso (2015), existe un trasvase de cierta apropiación de las maneras de diseñar que es interpretado y manipulado para producir un objeto de arte. En las enseñanzas de las Bellas Artes el fin que se persigue es la producción artística, pero cada vez más, existe mayor presencia en ella de elementos del mundo del diseño. En este sentido, cabe la posibilidad de aceptar que también puede darse la lectura contraria y que en los procesos de definición y generación del diseño existan cada vez más pretextos y maneras de proceder artísticos.

Sin olvidar que el diseño posee una finalidad estética, pero, sobre todo, funcional, el abordaje de sus procedimientos de enseñanzaaprendizaje desde las artes ofrece al futuro diseñador la posibilidad de entender mejor la relación de sus productos y sus usuarios, punto en el que la interactividad toma especial relevancia. El artista y diseñador contemporáneos, poseen un especial interés en que sus mensajes estén acompañados de una experiencia estética satisfactoria, que se haga posible gracias a la simplificación y naturalización de los canales de comunicación en los que se inscriban sus mensajes, del mismo modo que le suceda a los entornos, aplicaciones o artefactos que los vehiculen. Un ejemplo de ello sería la tecnología que posibilita la experiencia de realidad virtual, permitiendo que el usuario se mueva cada vez con más libertad sin que los cables o el volumen de los útiles que la permiten le recuerden permanentemente que lo que tiene delante no es real.

El Museo Virtual ESIT aparece ante el espectador como un espacio de realidad virtual. Para que sea posible, se utilizan diversas tecnologías de computación para crear un mundo tridimensional ficticio que pueda ser explorado y manipulado por un usuario, proporcionando la sensación de estar en dicho mundo (Stricland, 2018). Una importante cualidad de los principales tipos de entornos virtuales es su accesibilidad en cualquier momento y desde cualquier dispositivo de computación, de escritorio/portátil o móvil. Pero, si además tenemos presente la posibilidad de hacer más real si cabe la experiencia de inmersión en estos espacios interactivos a la hora, por ejemplo, de vincular el entorno exterior del espacio a las horas del día, tendremos una experiencia memorable que afiance esa sensación de "real" para sus visitantes. 


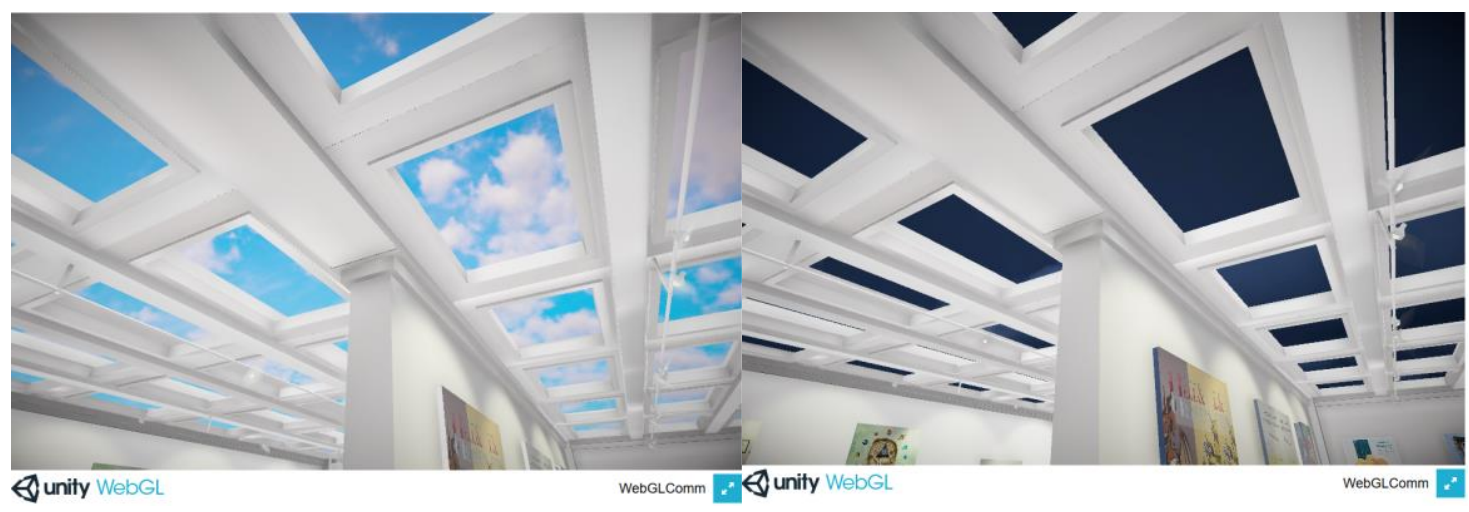

Figura 4 y 5: El lucernario del techo del Museo Virtual ESIT, ofrece una visión del cielo exterior que cambia del día a la noche según a la hora a la que se acceda (en horario GMT+1), 2018. Proyecto Museo Virtual ESIT.

\section{METODOLOGÍA}

\section{El arte de mostrar el diseño: El Learning by Doing en STEAM}

La Bauhaus (1919-1933) instauró por primera vez los fundamentos académicos, normativas, etc. del diseño. En aquel momento se trataba de formular una reforma de las enseñanzas de las artes y oficios con objeto de mejorar el nivel de los talleres de muebles y artesanía, que estaban siendo amenazados por la industrialización. Lo que pretendía su programa era potencial la creatividad de los estudiantes y favoreciendo así el descubrimiento y la evaluación de los medios de expresión individuales. Así, los principales ejes de la enseñanza que allí se desarrollaba ennoblecía la relación de empatía del alumno con el mundo. Casi un siglo después de la fundación de esta Escuela, que sería la primera de diseño del S. XX convirtiéndose en el referente internacional más importante de su campo, y sin perder el espíritu de los grandes maestros de entonces, la integración del Arte en áreas de Ingeniería y Tecnología es una realidad para la adquisición de las competencias propias de las diversas formaciones en las que esta sinergia se produce.

En formaciones de diseño en niveles superiores, tanto la visita a exposiciones de artistas y museos, como la exposición de la obra del alumnado es una parte fundamental de la formación de los mismos. La educación de la mirada es fundamental, hay que aprender a ver y hay que aprender a mostrarse. En este paradigma la cultura visual es fundamental para el diseñador que deberá formarse tanto a través de libros, como de Internet que hoy en día supone un recurso prácticamente ilimitado y en constante renovación para todos los diseñadores que quieren aprender del trabajo de otros. También existen múltiples comunidades digitales en las que poder ver y mostrar diseños por lo que, hoy más que nunca, no se concibe la creación de ningún proyecto de diseño sin hacer antes una prospección profunda de referencias y recursos útiles para plantear cualquier propuesta.

En este punto, la importancia de los procesos es fundamental en cualquier proyecto de diseño, lo interesante no está sólo en el resultado final, pues sin duda alguna también lo está en el camino que el alumnado debe recorrer hasta llegar a crear esas creaciones finales que, de alguna forma, les representan. Después de tanto trabajo y esfuerzo ante un proyecto personal también debemos pensar en otra dimensión, la de exponer, no sólo sus trabajos, sino exponerse personalmente.

Exhibirse, mostrarse, abrirse a los demás, supone en cierta forma también un riesgo, pues conlleva inevitablemente quedar expuesto ante la crítica, aunque si antes hemos pasado por la autocrítica, los estudiantes estarán más familiarizados con el ejercicio de mostrar, y defender si llega el caso, lo que saben y pueden llegar a hacer. En este sentido, uno de los objetivos de STEAM es fomentar "el impulso de la integración del Arte+Diseño en la educación y formación técnica superior en un enfoque de aprendizaje a lo largo de la vida" (Amor, 2018).

En el futuro profesional del estudiante esto supondrá la esencia de su trabajo, ya que todo lo que realicen deberá dar una respuesta a un requerimiento y, por el camino, la formulación de opiniones del resto de personas implicadas sobre lo que está haciendo, será constante. Colocar al estudiante en supuestos reales como los que acontecen en este proyecto, no solo encaja con el paradigma formativo que presenta STEAM sino que también lo hace con la metodología pedagógica Learning by Doing (Ferández, Nuviala, Pérez, Grao y González, 2012) que adaptamos en este proyecto para que los estudiantes desarrollen sus habilidades en un contexto real haciendo hincapié en lo necesario que es contar con un ambiente controlado y simulado para poder trabajar en proyectos que sitúan a los estudiantes en el centro de su proceso de enseñanza-aprendizaje, basado en la adquisición de competencias, habilidades, capacidades y destrezas para demostrarse a sí mismos qué son capaces de hacer. 
En la formación tradicional, el rol del profesor se centraba en la enseñanza-emisión- transmisión de conocimientos, mientras que el rol del estudiante se centraba en la recepción y asimilación de conocimientos. Sin embargo, en el Espacio Europeo de Educación Superior (EEES) y por la introducción de las nuevas tecnologías de la información y la comunicación (NTIC), el profesor pasa a ser orientador y dinamizador del proceso de aprendizaje de los estudiantes, es decir, el profesor ha de proporcionar al estudiante los criterios necesarios para saber buscar, encontrar y seleccionar la información que necesita para convertirla en conocimiento. (Ferández, Nuviala, Pérez, Grao y González, 2012, p. 160)

Hoy en día, todos los estudiantes han de adquirir competencias digitales, humanísticas y sociales para ser parte de la sociedad del conocimiento y la información de cara a dar respuesta a las demandas del tejido productivo y social. Esto se hace posible cuando los docentes de diversas disciplinas dentro de sus formaciones trabajan en equipo, con la funcionalidad integrada que un proyecto de estas características requiere. Así, llegamos a un escenario en el que un PDI multidisciplinar (artistas, diseñadores e ingenieros) se complementa trabajando en equipo, posibilitando que la enseñanza y el aprendizaje a través de modelos STEAM y la divulgación de resultados de la actividad formativa de los estudiantes fruto de estos procesos, sean presentados desde una perspectiva contemporánea y coherente con el modelo de la universidad en la que se inscribe, cuyo lema es "La Universidad en Internet". El Museo Virtual ESIT desde la perspectiva STEAM en enseñanza universitaria, supone el marco idóneo para perseguir los siguientes objetivos de forma simultánea:

1. Acercar a los estudiantes al mundo profesional, en el que poner a prueba sus habilidades a la hora de presentarse a una convocatoria pública o concurso de cualquier entidad.

2. Hacer posible en este proyecto la colaboración del PDI del área de diseño encargado de desarrollarlo, con ingenieros informáticos e ingenieros de espacios interactivos para su materialización y mantenimiento técnico, en el aprendizaje y reciclaje permanente que todo docente universitario necesita.

\section{DESARROLLO}

EI PIE Museo Virtual ESIT comienza a materializarse a partir de una serie de bocetos en cuanto a la distribución del espacio y los puntos de luz necesarios.
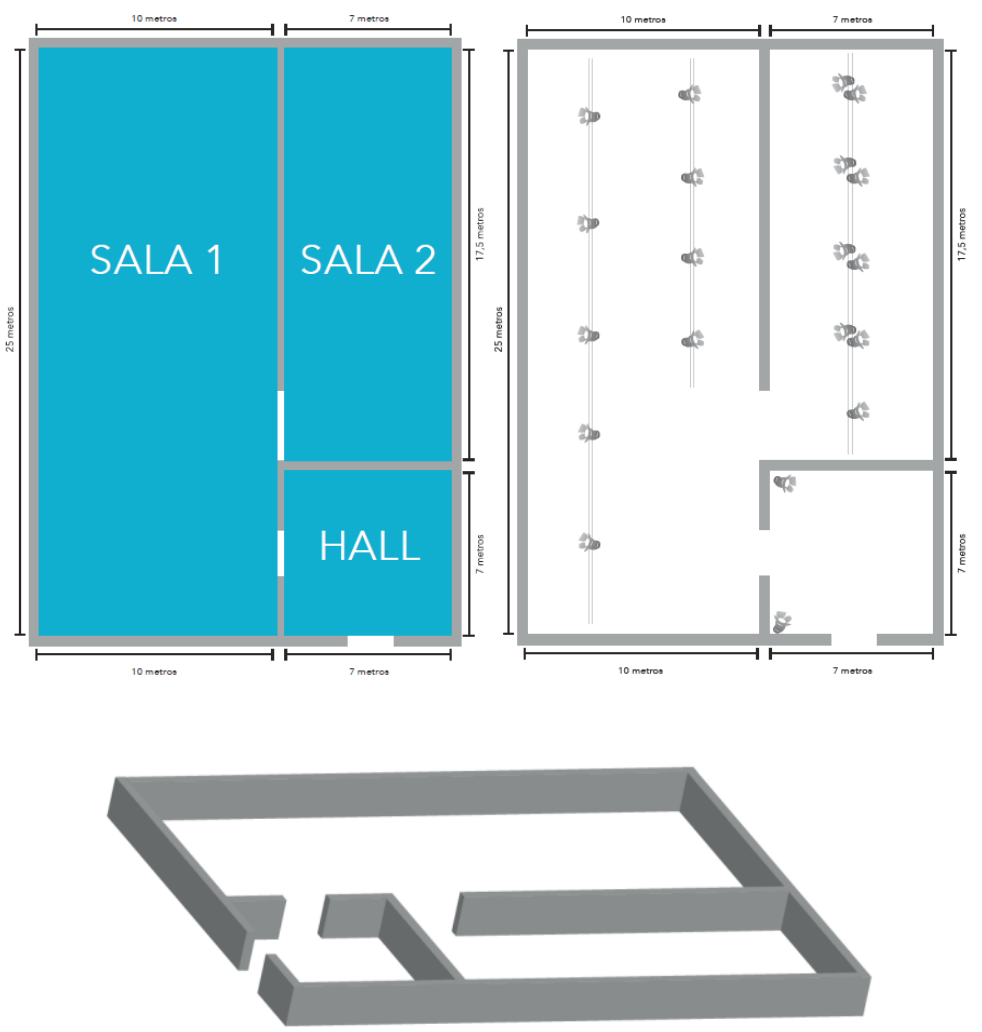

Figura 6, 7 y 8: Bocetos digitales del diseño de los espacios del Museo Virtual ESIT, 2018. Proyecto Museo Virtual ESIT. 
De Miguel Álvarez, Laura; Rúas Ruiz-de Infante, Jaime; Ríos Aguilar, Sergio; González Crespo, Rubén

Seguidamente, sobre el modelado, se valoran los acabados a aplicar en el prototipo para obtener un espacio limpio, amplio y elegante, que recuerde a la imagen mental que el visitante puede tener de un Museo.

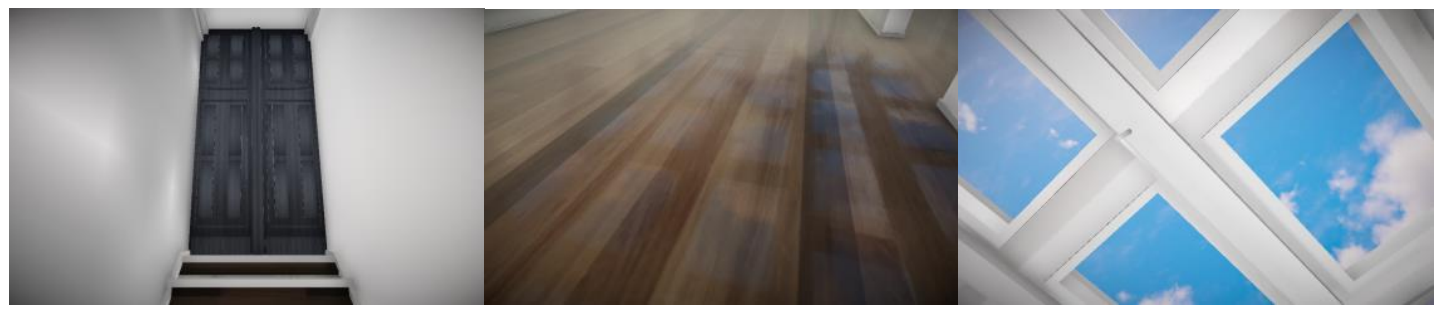

Figura 9, 10 y 11: Detalle de acabados de los espacios del Museo Virtual ESIT, 2018. Proyecto Museo Virtual ESIT.

Modelado el espacio y valorada la interacción en él, se sucede el lanzamiento de diversas convocatorias abiertas a todos los estudiantes del área de diseño de ESIT.

Estas convocatorias son presentadas a través de unas bases (como en cualquier actividad de difusión similar) que, genéricamente, poseen el siguiente contenido que se adapta según la especificidad de cada exposición:

- Disciplina: Todas las comprendidas en el Área de Diseño de ESIT.

- Temática: Para cada caso se establece una temática que es presentada de forma clara a los estudiantes para que puedan establecer sus soluciones. Por ejemplo, en la exposición que se realizó en colaboración con Isidro Ferrer, los estudiantes tenían que realizar una imagen sin texto (fotografía, ilustración, composición digital) a partir de la frase "La transformación digital la hacen personas creativas" (frase que tenía que servirles para inspirarse pero que no debía aparecer en la imagen).

- Premios: se especifica su naturaleza en las bases.

- Criterios de selección: aspectos a tener en cuenta para la valoración de las obras.

- Especificaciones técnicas de la solución requerida: Se aporta las dimensiones que debe tener la solución, el formato y la calidad mínima.

- Envío y documentación a entregar: Se solicitan los datos personales del participante y un permiso de cesión de derechos de difusión de obra debidamente cumplimentado y firmado, para el cual se les facilita un modelo.

- Fechas importantes: Se establece el calendario que los participantes deben tener en cuenta para las sesiones informativas, fecha límite de recepción de obras, fecha de inauguración y fallo del jurado.

Recibidas las obras y teniendo como referencia los criterios de selección que aparecían en las bases, la comisión que gestiona el Museo valora qué obras van a ser finalmente expuestas y, de ellas, cuáles serán las ganadoras de los premios establecidos en cada convocatoria. La comisión a la que se hace referencia, está formada por los docentes de ESIT que gestionan el proyecto, el director de la Escuela Superior de Ingeniería y Tecnología y, de haberse contemplado en las bases alguna colaboración con empresa o profesional del sector del diseño, ese agente también será parte de la comisión para la selección de las obras y veredicto final para el reparto de los premios.

El siguiente paso es repartir las obras en las salas de exposiciones. Para ello se toman en cuenta cuestiones relacionadas con las dimensiones, el contenido (sobre todo en cuanto al color y el mensaje) y la calidad técnica del arte final. Pero antes se realiza un fotomontaje por cada exposición planificada, para facilitar la colocación final de las obras con el gestor.

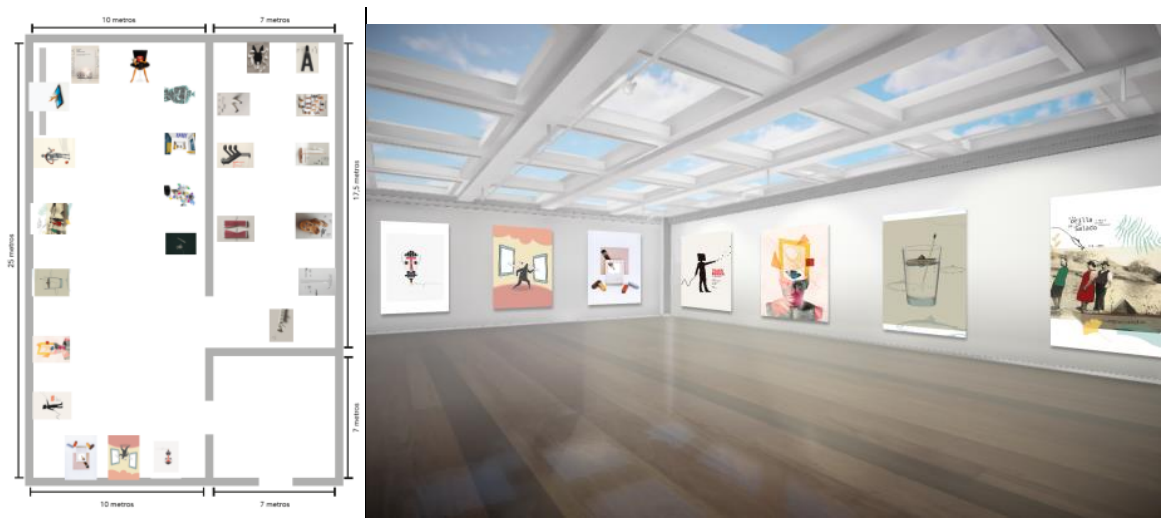

Figura 12, 13 y 14: Fotomontajes de las obras seleccionadas en los espacios del Museo Virtual ESIT, 2019. Proyecto Museo Virtual ESIT. 
De Miguel Álvarez, Laura; Rúas Ruiz-de Infante, Jaime; Ríos Aguilar, Sergio; González Crespo, Rubén El Museo Virtual ESIT como recurso para la enseñanza-aprendizaje en áreas de Diseño y Tecnología IV Congreso INTERNACIONAL DE INVESTIGACIÓN EN ARTES VISUALES ANIAV 2019 IMAGEN [N] VISIBLE]

http://dx.doi.org/10.4995/ANIAV.2019.9000

Una vez hecho el reparto, se colocan las obras con el gestor de contenidos de Unity (que es la herramienta elegida para el modelado del espacio), y las cartelas y paneles pertinentes. En ese momento, el espacio está listo para abrirse el público.

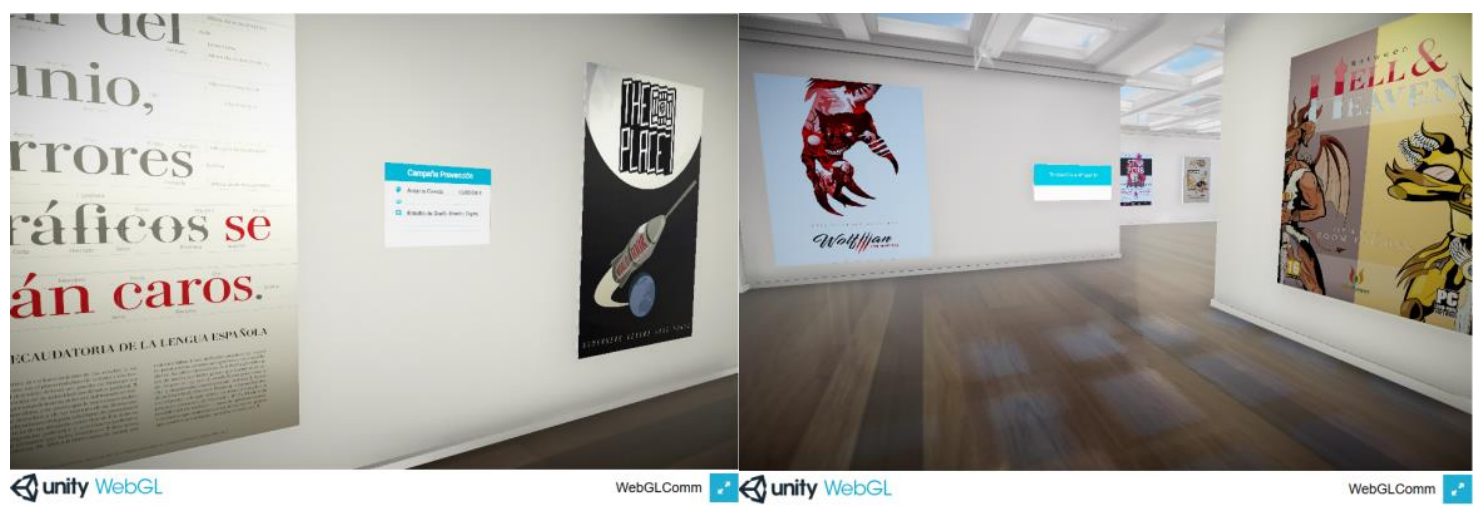

Figura 15 y 16: captura de la exposición del Museo Virtual ESIT, 2018. Proyecto Museo Virtual ESIT.

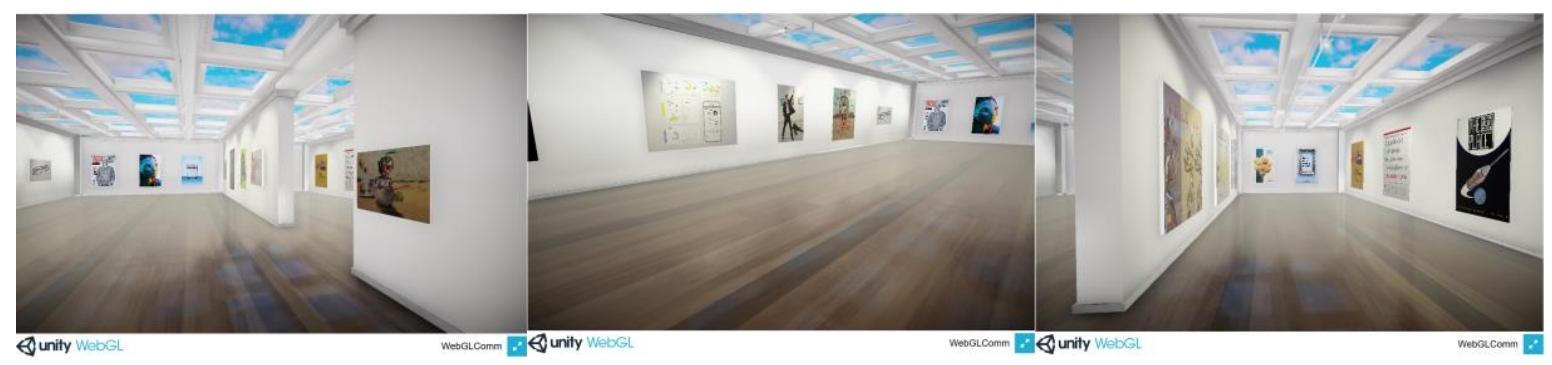

Figura 17, 18 y 19: Capturas de pantalla del espacio expositivo del Museo Virtual ESIT, 2018. Proyecto Museo Virtual ESIT.

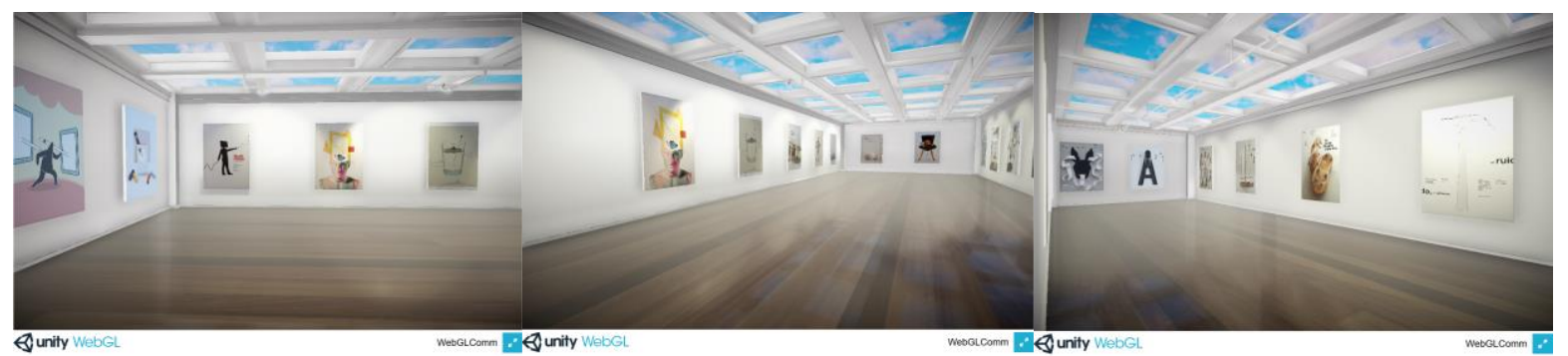

Figura 20, 21 y 22: Capturas de pantalla del espacio expositivo del Museo Virtual ESIT, 2019. Proyecto Museo Virtual ESIT.

\section{CONCLUSIONES}

Un proyecto como el presentado en este artículo, que continua vivo y proyectando su tercera exposición, favorece que los estudiantes de áreas creativas se acerquen a posibles escenarios que podrán encontrase en su futuro profesional, en los que tendrán que poner a prueba sus habilidades a la hora de presentar lo que son capaces de hacer frente a un requerimiento determinado. Pero también son proyectos que posibilitan el trabajo en equipo de docentes provenientes de áreas de diseño con ingenieros informáticos y de espacios interactivos en su desarrollo y mantenimiento.

Consideramos altamente positivo el hecho de que estos proyectos se produzcan en un momento en el que la universidad y la forma en la que los alumnos acceden al conocimiento, donde el docente se convierte a veces en cliente, otras veces en director de arte o en comisario, los estudiantes han de ser capaces de aprender a través de sus propias acciones, entre otras, han de conectarse con otros profesionales y aprender haciendo y mostrando.

El reto que la comunidad educativa tiene delante es el de trabajar juntos favoreciendo el florecimiento del conocimiento en todas direcciones. Los docentes ya no pueden ser meros transmisores de su saber, deben estar preparados para los retos que plantea la 
De Miguel Álvarez, Laura; Rúas Ruiz-de Infante, Jaime; Ríos Aguilar, Sergio; González Crespo, Rubén

El Museo Virtual ESIT como recurso para la enseñanza-aprendizaje en áreas de Diseño y Tecnología

IV Congreso INTERNACIONAL DE INVESTIGACIÓN EN ARTES VISUALES ANIAV 2019

IMAGEN [N] VISIBLE]

http://dx.doi.org/10.4995/ANIAV.2019.9000

nueva sociedad del conocimiento en la que el acceso a la información every moment, every where (cualquier momento, cualquier lugar) es un hecho cotidiano que los desplaza de la posición en la que desde el aprendizaje tradicional se les había colocado.

El desarrollo de proyectos conjuntos, trabajando desde distintos puntos de vista que enriquezcan el trabajo global, provoca que el PDI actúe desde esa bidimensionalidad de docentes investigadores para/con sus estudiantes en la materialización de un proyecto que ocupa y motiva a ambos. Los estudiantes, han de ser capaces de no atender a sus prejuicios o inseguridades a la hora materializar lo que son capaces de hacer. $Y$, en el mismo sentido, los docentes de áreas de diseño deben seguir trabajando para acercar a sus estudiantes la realidad que se van a encontrar cuando se sumerjan en el ámbito profesional. Para este reto, el marco que propicia el desarrollo de propuestas bajo la denominación STEAM en formación universitaria en áreas de Ingeniería y Tecnología, suponen ser idóneo en la construcción de proyectos estimulantes y productivos para la comunidad educativa en su conjunto.

\section{FUENTES REFERENCIALES}

Amor Braco, E. (2018). De STEM a STEAM: mucho más que la interacción del arte y la ciencia. En Educaweb. Recuperado de https://www.educaweb.com/noticia/2018/04/04/stem-steam-mucho-mas-interaccion-arte-ciencia-16384/

Annetta, L.A., Folta, E., and Klesath, M. (2010). V-Learning: Distance Education in the 21st Century through 3D Virtual Learning Environments. Ed. Springer

Fernández, A., Nuviala, A., Pérez, R., Grao, A. González, J.J. (2012). Estudio Comparativo entre una metodología de aprendizaje tradicional respecto a una metodología de aprendizaje basada en el 'Learning by doing' para la consecución de competencias específicas. Revista UPO INNOVA, 1, 159-169. Universidad Pablo de Olavide. Sevilla. Recuperado de https://rio.upo.es/xmlui/handle/10433/1762

Haro González, S. y Alonso Calero, J.M. (2015). Estrategias docentes en la interacción Arte / Diseño en la enseñanza superior de las Bellas Artes. Actas de Diseño, 19, 202-207. Facultad de Diseño y Comunicación. Universidad de Palermo. Recuperado de http://fido.palermo.edu/servicios dyc/publicacionesdc/archivos/541 libro.pdf

HORIZON 2020 WORK PROGRAMME 2014 - 2015 16. Science with and for Society Revised. (European Commission Decision C (2015) 2453 of 17 April 2015). p. 6. Recuperado de https://ec.europa.eu/info/funding-tenders/opportunities/portal/screen/opportunities/projectsresults; programCode $=\mathrm{H} 2020$

Johanes, C. (2018). ¿Qué es? (STEAM). Recurso interactivo de la Fundación Bancaria "la Caixa". El taller interactivo S.L. Recuperado de https://www.educaixa.com/microsites/steam/steam es/recursos aux/

Manesh, H. y Schaefer, D. (2010). Virtual Learning Environments for Manufacturing. https://doi.org/10.4018/978-1-61520-619-3.ch006

Muñoz, J. (2015). STEM, STEAM... Pero ¿qqué es? Recuperado de http://odite.ciberespiral.org/comunidad/ODITE/recurso/stem-steampero-eso-que-es/58713dbd-414c-40eb-9643-5dee56f191d3

Stricland, J. (2018). How Virtual Reality Works. Recuperado de https://electronics.howstuffworks.com/gadgets/other-gadgets/virtualreality.htm 